\section{Development of the first edition of African treatment guidelines for common bacteri- al infections and syndromes}

\author{
Jessica Craig, ${ }^{1}$ Isabel Frost, ${ }^{2-4}$ Aditi \\ Sriram, ${ }^{2}$ James Nuttall, ${ }^{5}$ Geetanjali \\ Kapoor, ${ }^{2}$ Yewande Alimi, ${ }^{6}$ \\ Jay K. Varma ${ }^{6,7}$ \\ ${ }^{1}$ Center for Disease Dynamics, \\ Economics and Policy, Washington, DC, \\ USA; ${ }^{2}$ Center for Disease Dynamics, \\ Economics and Policy, New Delhi, India; \\ ${ }^{3}$ Imperial College London, London, UK; \\ ${ }^{4}$ Amity University, Noida, India; ${ }^{5}$ Red \\ Cross War Memorial Children's \\ Hospital and University of Cape Town, \\ Cape Town, South Africa; ${ }^{6}$ Africa \\ Centres for Disease Control and \\ Prevention, Addis Ababa, Ethiopia; \\ ${ }^{7}$ Weill Cornell Medicine, Department of \\ Population Health Sciences, New York, \\ USA
}

\begin{abstract}
Standard treatment guidelines (STGs) are an important tool for ensuring high quality clinical care and prudent antimicrobial use (AMU) and stewardship (AMS). In 2018, African Union (AU) member state representatives recognized the lack of STGs as a barrier to AMS at national and facility levels. Previous research reported that only 17 of $55(31 \%)$ member states had STGs that provided disease- or pathogen-specific antimicrobial treatment recommendations, excluding those that covered only treatment of HIV, malaria, and tuberculosis). The Africa Centres for Disease Control and Prevention convened expert panels to develop first edition antibiotic treatment guidelines for priority infectious diseases and clinical syndromes for pediatric and adult patient populations in Africa. The purpose of the guidelines is to provide healthcare workers with treatment guidance by harmonising existing national STGs, filling gaps where existing STGs are not available, and serving as a model for future guidelines. Two expert panels of 28 total clinicians, pharmacists, and other relevant stakeholders from 14 AU member states representing each continental region convened to develop consensus treatment recommendations for select priority bacterial infections and clinical syndromes. In developing recommendations, the panels considered treatment recommendations from existing STGs, drug availability, clinical experience,
\end{abstract}

and available antimicrobial resistance data. The guidelines underwent an external review process where clinical stakeholders who did not serve on either panel were invited to submit feedback prior to their publication. The guidelines provide empiric antibiotic therapy guidelines - including drug selection, route of administration, formulation, dosage, and therapy duration and principles of stewardship for 28 bacterial infections or clinical syndromes. The first edition guidelines for the treatment of common infectious diseases and clinical syndromes in Africa aims to improve clinical treatment and antimicrobial stewardship and will serve as a template for future regional guidelines.

\section{Introduction}

Antimicrobial resistance (AMR) poses a significant and rapidly emerging public health challenge in Africa and around the world. ${ }^{1-3}$ In many countries, drug-resistant infections have been associated with longer hospital stays, increased morbidity and mortality, and higher healthcare costs. ${ }^{4-6}$ Despite the challenge, antimicrobial stewardship (AMS) solutions are not widespread. ${ }^{7-10}$

Standard treatment guidelines (STGs) are one important tool in ensuring high quality clinical care and prudent antimicrobial use, alongside other AMS and educational efforts. ${ }^{11,12}$ STGs, when developed and used properly, help clinicians prescribe the right antimicrobial for the right condition, ensuring that the selection, dosage, and duration align with local epidemiology, AMR prevalence, laboratory and other diagnostic capacities, and pharmaceutical availability. A 2019 study conducted in Norway found that adherence to national antibiotic guidelines was associated with favorable patient outcomes, including reduced mortality rates and length of hospital stay among inpatients. ${ }^{13}$

In 2018, African Union (AU) member state representatives and stakeholders recognized the lack of STGs as a barrier to AMR mitigation and AMS efforts and identified the development of STGs as a priority activity. A previous review of existing STGs in AU member states identified only 28 relevant guidelines (excluding those that covered only treatment of HIV, malaria, and tuberculosis) from 17 of 55 (31\%) member states that provided disease- or pathogenspecific antimicrobial treatment recommendations including drug selection, dosage, and therapy duration. ${ }^{14}$ Of those, only 15 guidelines from 10 countries were developed or updated in the past five years. No
Correspondence: Jessica Craig, 962 Wayne Ave, Suite 530, Silver Spring, Maryland 20910, USA.

Tel: +1.202.939.3300.

E-mail: craig@cddep.org

Key words: Infectious diseases, Bacterial infections, Clinical treatment guidelines, Antimicrobial stewardship.

Acknowledgements: The authors wish to thank Marc Mendelson for his role as a panel chair, all panel participants who developed the guidelines and reviewed the methodologies, and external reviewers who reviewed the guidelines.

Contributions: YA and JK conceived of the project; JC, IF, AS, GK, YA, and JK served on the steering committee and developed the methodologies. JN served as a panel chair.

Conflict of interest: The authors declare no conflicts of interest.

Funding: This work was supported by the Training Programs in Epidemiology and Public Health Interventions Network (TEPHINET).

Received for publication: 12 August 2021.

Revision received: 14 December 2021.

Accepted for publication: 19 December 2021.

This work is licensed under a Creative Commons Attribution NonCommercial 4.0 License (CC BY-NC 4.0).

CC Copyright: the Author(s), 2021

Licensee PAGEPress, Italy

Journal of Public Health in Africa 2021; 12:2009 doi:10.4081/jphia.2021.2009

identified guideline described their methodologies or adhered to standardized methodologies such as the Grading of Recommendations Assessment, Development and Evaluation (GRADE) approach, and few stated that they were based on local infectious disease epidemiology or AMR data. A comparison of the antimicrobial selection, dosage, and therapeutic duration set forth by the identified guidelines found wide variation including instances where for certain infections some guidelines recommended antimicrobial therapy and others discouraged antimicrobial therapy altogether.

Given the lack of STGs in AU member states and to promote better quality healthcare and AMS across the continent, we convened expert panels to develop a first edition of African antibiotic treatment guidelines for priority infectious diseases and 
clinical syndromes for pediatric and adult patient populations.

\section{Materials and methods}

The development of the guidelines was led by a steering committee composed of six physicians, epidemiologists, and public health researchers from the Africa Centres for Disease Control and Prevention (CDC) and the Center for Disease Dynamics, Economics \& Policy (CDDEP). The steering committee was responsible for managing the overall guidelines development project, assembling the expert panels, drafting and finalizing the guidelines, coordinating internal and external review, and disseminating the guidelines. The steering committee also drafted methodologies for the guideline development project including for convening an expert panel review, generating consensus treatment recommendations, and disseminating the findings. Finally, the committee conducted reviews for existing AMR data and national STGs from AU member states and synthesized the data into reports for expert panel review and to inform aspects of guidelines development, such as drug and formulation availability, preferred treatment paradigms in various countries, and drug selection in settings with high AMR prevalence rates. Results of both reviews have been submitted for publication. ${ }^{14,15}$ Briefly, the paucity of locally derived data about AMR prevalence meant that such data had little impact on panel decision-making.

\section{Convening guidelines development groups}

The steering committee invited physicians, pharmacists, and other relevant experts involved in diagnosing and treating infectious diseases and/or prescribing antimicrobials to participate in one of two guidelines development panels. In selecting participants, the steering committee strove to represent each African region and balance gender.

The first panel consisted of nine expert clinicians representing each African region (per the United Nations) and seven countries (Northern: Egypt; Southern: South Africa; Eastern: Kenya, Zambia; Middle: Republic of the Congo; Western: Ghana, Senegal) and convened in August 2019 at AU headquarters in Addis Ababa, Ethiopia to develop treatment recommendations for adult patient populations. ${ }^{16}$ The second panel, composed of 19 experts representing each African region and 12 countries (Northern: Egypt; Southern: South Africa; Eastern: Ethiopia, Kenya, Mozambique,
Tanzania, Uganda; Middle: Gabon; Western: Gambia, Ghana, Nigeria, Senegal), convened in October 2020 via virtual webinar (due to the SARS-CoV-2 pandemic) to develop treatment recommendations for pediatric patient populations.

The panels reviewed and finalized the methodology prepared by the steering committee and results of the committee's analysis of existing AMR data and STGs in Africa. The panels also established a standardized format for presenting the treatment recommendations and clinical guidance. The panels were tasked with selecting infections and clinical syndromes for inclusion in the first edition guidelines. For each selected infection or syndrome, each panel set forth treatment recommendations which included antibiotic selection, dosage, and duration and principles of stewardship and other clinical notes, as applicable. Preferred and alternative antibiotic choices were discussed and provided, as needed, as were treatment recommendations for special cases such as in case of confirmed allergy or medical contra-indication. The treatment recommendations were developed through round-table discussions, led by the panel chair, and were finalized by consensus. Draft guidelines and methodologies were developed and disseminated to panelists for review and approval prior to external review.

\section{Conducting an external review}

Once final drafts of the guidelines were developed, we posted them on the Africa $\mathrm{CDC}$ website and used social media and existing e-mail list servs to request comment from people with relevant expertise. Additional clinicians based in Africa were identified through a web search and literature review and were sent targeted e-mails inviting them to review the guidelines. At least two clinicians from each African country were contacted, while additional experts were contacted in under-represented countries and regions including central and francophone West African countries.

A total of 22 external reviewers representing 11 African countries (Southern: South Africa; Eastern: Ethiopia, Kenya, Mauritius, Rwanda, Tanzania, Uganda; Zimbabwe; Middle: Cameroon; Western: Gambia, Nigeria) and 26 organizations or institutions including Mount Kenya University, University of Ibadan (Nigeria), the Ministry of Health and Wellness of Mauritius, University of Global Health Equity (Rwanda and Uganda), the Association of Zimbabwe Pharmacists Abroad, and Médecins Sans Frontiers provided feedback on the draft guidelines. The steering committee reviewed and compiled the input and each panel chairperson provided final decisions on the inclusion or exclusion of each proposed edit, addition, or deletion.

The final guidelines were compiled into a single report and into two quick reference guides, one each for adult and pediatric patient populations, and translated into all official AU languages: Arabic, French, Portuguese, and Spanish. ${ }^{17-19}$ A user-friendly website displaying the treatment recommendations and other information about the guidelines development process is now available at https://africacdc.org/africanantibiotic-treatment-guidelines-for-common-bacterial-infections-and-syndromes/ and at https://africaguidelines.cddep.org/; the guidelines report and quick reference guides are also available for download.

\section{Ethical considerations}

Ethics approval was not required for this study as no human subjects research was conducted, and all data utilized was in the public domain. Panelists were required to report any potential conflicts of interest including intellectual property and financial ties to pharmaceutical companies before participation.

\section{Results}

\section{Clinical diseases and syndromes covered}

The first-convened panel agreed to focus on empiric antibiotic therapy guidelines, because appropriate specimen collection and diagnostic testing to identify causative pathogens is not universally available in Africa. Therefore, the panel sought to provide appropriate antibiotic choice(s) to treat an infection for which a specific etiological diagnosis has not been made, targeting the most likely pathogen(s) with the narrowest-spectrum, single antibiotic whenever possible while also accounting for the likelihood of antibiotic resistance, potential medical contraindications (i.e. allergies, pregnancy) and target tissue penetration.

The panel elected to limit the scope of the first edition guidelines to include only common bacterial infections and syndromes; therefore, this scope was maintained during the second panel convening. The guidelines set forth recommended antibiotic therapies for 28 infections or clinical syndromes; 11 are common to both adult and pediatric patient populations including acute, community-acquired bacterial meningitis; acute otitis media; bacterial pharyngotonsillitis; dental abscess; infective endocarditis; acute diarrheal dis- 
Table 1. Example guideline for the treatment of suspected bacterial meningitis (community-acquired) for pediatric patient groups.

\begin{tabular}{|c|c|c|c|}
\hline \multicolumn{4}{|c|}{ Preferred antibiotic choice, neonate } \\
\hline $\begin{array}{l}\text { Combination therapy with: } \\
\text { Cefotaxime (IV) } \\
\text { PLUS } \\
\text { Ampicillin (IV) }\end{array}$ & $\begin{array}{l}\text { Cefotaxime- Powder for } \\
\text { injection: } 250 \text { or } 500 \mathrm{mg} \text { per vial } \\
\text { (as sodium salt) } \\
\text { Ampicillin- Powder for injection: } \\
500 \mathrm{mg}, 1 \mathrm{~g} \text { (as sodium salt) in vial }\end{array}$ & $\begin{array}{l}\text {-First week of life ( } 7 \text { days or less): } \\
50 \mathrm{mg} / \mathrm{kg} / \mathrm{dose} 12 \text { hourly } \\
-8-20 \text { days: } 50 \mathrm{mg} / \mathrm{kg} / \mathrm{dose} 8 \text { hourly } \\
-21 \text { days \& older: } 50 \mathrm{mg} / \mathrm{kg} / \text { dose } 6 \text { hourly } \\
\text {-First week of life }(7 \text { days or less): } \\
100 \mathrm{mg} / \mathrm{kg} / \text { dose } 8 \text { hourly } \\
-8 \text { days of age and older: } \\
100 \mathrm{mg} / \mathrm{kg} / \text { dose } 6 \text { hourly }\end{array}$ & $\begin{array}{l}\text { Treat with ampicillin (for Listeria coverage) until CSF } \\
\text { culture results confirm etiology. } \\
\text { If CSF culture is not available, treat with } \\
\text { cefotaxime plus ampicillin for } 14 \text { - } 21 \text { days. }\end{array}$ \\
\hline \multicolumn{4}{|c|}{ If cefotaxime is not available, use } \\
\hline $\begin{array}{l}\text { Combination therapy with: } \\
\text { Ceftriaxone (IV) } \\
\text { PLUS } \\
\text { Ampicillin (IV) } \\
\text { (Except in neonates with }\end{array}$ & $\begin{array}{l}\text { Powder for injection: } 250 \mathrm{mg} \text {; } \\
1 \mathrm{~g} \text { (as sodium salt) in vial } \\
\text { Ampicillin- Powder for } \\
\text { injection: } 500 \mathrm{mg} \text {, } \\
1 \mathrm{~g} \text { (as sodium salt) in vial }\end{array}$ & $\begin{array}{l}50 \mathrm{mg} / \mathrm{kg} / \mathrm{dose} 12 \text { hourly } \\
\text {-First week of life ( } 7 \text { days or less): } \\
100 \mathrm{mg} / \mathrm{kg} / \text { dose } 8 \text { hourly } \\
-8 \text { days of age and older: }\end{array}$ & $\begin{array}{l}\text { Treat with ampicillin (for Listeria coverage) } \\
\text { until CSF culture results confirm etiology. If CSF } \\
\text { culture is not available, treat with ceftriaxone plus } \\
\text { ampicillin for 14-21 days. }\end{array}$ \\
\hline
\end{tabular}

(Except in neonates with $1 \mathrm{~g}$ (as sodium salt) in vial

jaundice and neonates

-8 days of age and older:

$100 \mathrm{mg} / \mathrm{kg} /$ dose 6 hourly

receiving calcium-containing

IV fluids)

\begin{tabular}{|c|c|c|c|}
\hline & Preferred antibiotic ch & , infant (older than $28 \mathrm{da}$ & ind adolescent \\
\hline Drug(s) & Formulation & Dosage & Duration \\
\hline Ceftriaxone (IV) & $\begin{array}{l}\text { Powder for injection: } 250 \mathrm{mg} \text {; } \\
1 \mathrm{~g} \text { (as sodium salt) in vial }\end{array}$ & $\begin{array}{l}50 \mathrm{mg} / \mathrm{kg} / \text { dose } 12 \text { hourly, } \\
\text { maximum dose } 2 \mathrm{~g} 12 \text { hourly }\end{array}$ & 10 - 14 days \\
\hline
\end{tabular}

$\begin{array}{lll}\text { Ampicillin (IV) } & \text { Powder for injection: } 500 \mathrm{mg} ; & 50 \mathrm{mg} / \mathrm{kg} / \mathrm{dose} 6 \text { hourly, maximum dose: } \quad 10 \text { - } 14 \text { days } \\ & \lg \text { (as sodium salt) in vial } & 2 \mathrm{~g} 6 \text { hourly }\end{array}$

Clinical definition: Inflammation of meninges of the brain and spinal cord. Clinical features may be non-specific in neonates and young infants (e.g. poor feeding, apathy, jaundice, apnoea, full fontanelle, fever, hypothermia) and in older infants may include irritability, drowsiness, poor feeding, high fever, and/or vomiting. Older children may present similarly to adults with headache, fever, photophobia, vomiting, neck stiffness, and/or altered level of consciousness. Common bacterial pathogens in neonates and young infants include Streptococcus agalactiae (Group B Streptococcus), E. coli, Klebsiella species, L. monocytogenes, and in older infants and children: S. pneumoniae, H. influenzae, and N. meningitidis. Principles of Stewardship: A) Acute meningitis may be caused by a range of pathogens, some of which are not bacteria. Microbiologic diagnosis, including CSF gram stain/microscopy, bacterial culture and AST should be obtained as soon as possible, if available, as this may allow empiric antibiotic treatment to be adjusted to target the specific pathogen identified and inform the duration of treatment. In the absence of a positive CSF culture or PCR result, a positive blood culture result together with a CSF cell count and chemistry suggestive of bacterial meningitis may be useful in guiding antibiotic selection and duration of treatment. Although guidelines differ in treatment duration recommendations for specific pathogens, a general recommendation for uncomplicated meningitis is Gram negative organisms and Listeria 21 days, Group B Streptococcus 14-21 days, S. pneumoniae 10-14 days, H. influenzae 7-10 days, N. meningitidis 5-7 days. B) In patients with a positive CSF culture, repeat lumbar puncture 24-48 hours after initiation of antimicrobial treatment to document CSF sterilization is useful (particularly in neonates) as delayed sterilization may be an indication of complications such as a purulent focus requiring intervention, or antibiotic resistance. C) If CSF is obtained and is not consistent with meningitis (e.g. absence of cells and normal chemistry), antibiotics should be stopped or adjusted depending on whether an alternative diagnosis has been reached. D) Consider diagnostic tests for tuberculous and cryptococcal meningitis, particularly in high HIV-burden areas. Other Notes: A) Complications include subdural empyema and brain abscess which may require neurosurgical intervention in addition to treatment with the above-mentioned antimicrobial therapy. B) In children and adolescents with a ventriculoperitoneal (VP) shunt presenting with meningitis, seek expert opinion and refer patient to a specialist where possible.

Table 2. Example guideline for the treatment of dental abscess (including gingivitis) for adult patient groups.

\begin{tabular}{|c|c|c|c|}
\hline Drug(s) & Formulation & $\begin{array}{l}\text { d antibiotic choice }(s) \\
\text { Dosage }\end{array}$ & Duration \\
\hline Amoxicillin-clavulanic acid (PO) & $\begin{array}{l}\text { Oral liquid: } 125 \mathrm{mg} \text { amoxicillin } \\
+31.25 \mathrm{mg} \text { clavulanic acid/5 mL } \\
\text { AND } 250 \mathrm{mg} \text { amoxicillin + }\end{array}$ & $\begin{array}{l}500 \text { mg of amoxicillin } \\
\text { component } 8 \text { hourly }\end{array}$ & $\begin{array}{l}3 \text { days if adequate source control, } \\
\text { or } 5 \text { days if not }\end{array}$ \\
\hline
\end{tabular}

$62.5 \mathrm{mg}$ clavulanic acid $/ 5 \mathrm{~mL}$;

Tablet: 500 mg amoxicillin (as trihydrate) + $125 \mathrm{mg}$ clavulanic acid (as potassium salt)

Phenoxymethyl-penicillin (penicillin V) (PO)

Powder for oral liquid: $250 \mathrm{mg} \quad 500 \mathrm{mg} 6$ hourly

3 days if adequate source control,

(as potassium salt) $/ 5 \mathrm{~mL}$; Tablet: 2

or 5 days if not

$50 \mathrm{mg}$ (as potassium salt)

\section{In case of confirmed drug allergy or medical contraindication}

Combination therapy with: Azithromycin (PO)

PLUS

Metronidazole (PO)
Azithromycin- Capsule: $250 \mathrm{mg}$; $500 \mathrm{mg}$ (anhydrous); Oral liquid: $200 \mathrm{mg} / 5 \mathrm{~mL}$ Metronidazole- Oral liquid: $200 \mathrm{mg}$ (as benzoate) $/ 5 \mathrm{~mL}$; Tablet: $200 \mathrm{mg}$ to $500 \mathrm{mg}$

Clinical definition: Tooth infections from cavities, gingivitis, and periodontitis. Common symptoms include severe pain, tooth sensitivity, and inflammation of the face and gums. Most infections are polymicrobial and include anaerobic bacteria. Principles of Stewardship: A) Dental abscess requires surgical drainage, not just antibiotics. B) If the abscess is drained and the patient is improving, consider stopping antibiotics after 3 days of treatment. C) Although gingivitis is a risk factor for dental abscess, only acute necrotizing gingivitis should be treated with antibiotics. D) For gingivitis without necrosis or abscess, do not treat with antibiotics. Other Notes: For acute necrotizing gingivitis: A) Treat with clindamycin [Dosage: Capsule: $150 \mathrm{mg}$ (as hydrochloride); Injection: $150 \mathrm{mg}$ (as phosphate)/mL; Oral liquid: $75 \mathrm{mg} / 5 \mathrm{~mL}$ (as palmitate)] for $3 \mathrm{days}$. B) For cases of acute necrotizing gingivitis associated with malnutrition, treat with vitamins. ${ }^{17}$ 
ease; community-acquired complicated intra-abdominal infection; typhoid/enteric fever; urinary tract infection; skin and soft tissue infection; and acute osteomyelitis/ septic arthritis. ${ }^{17}$ Treatment recommendations for acute bronchitis; acute exacerbation of chronic obstructive pulmonary disease (COPD); acute pyelonephritis; and acute, mild to moderate uncomplicated prostatitis are provided for adult patient populations only while recommendations for the treatment of acute purulent neonatal conjunctivitis, suspected acute bacterial sinusitis, acute rheumatic fever, syphilis, tetanus, and possible serious bacterial infection in infants are only provided for neonatal and pediatric patient populations. For adult patients, there are three separate guidelines for pneumonia including communityacquired, mild to moderate disease in ambulatory outpatients; community-acquired severe disease in hospitalized patients; and hospital-acquired (nosocomial) pneumonia. The pediatric guideline includes two treatment recommendations for communityacquired, acute lower respiratory tract infection, one for mild to moderate disease in ambulatory patients and another for severe disease in inpatients.

\section{Components of the guidelines}

The guidelines include an introductory section with general principles of stewardship and instructions for guideline use. Pediatric and adult treatment recommendations are presented in separate sections and are organized by the following body system or grouping classifications: Central nervous system; head, eye, ear, nose, and throat; cardiac; respiratory; gastrointestinal; genitourinary; skin, soft tissue, bone and joints; and bloodstream. Pediatric treatment guidelines including those for neonates, children, and adolescents are presented together with subheadings indicating their applicability to each of the sub-patient populations, as applicable (Table 1). Neonatal and pediatric age groups are defined as follows: Neonateless than 28 days old or if born prematurely, less than 42 weeks corrected gestational age; Infant- less than 1 year of age; Childless than 10 years of age; and adolescent- 10 to 19 years of age.

Treatment guidelines for each bacterial infection or syndrome are presented with the following information, as applicable: Clinical definition including common symptoms and causative microorganisms, preferred and alternative antibiotic choices, antibiotic choice in case of confirmed drug allergy or medical contraindication or in other specific circumstances, principles of stewardship, and other clinical notes (Table 2). Antibiotic choices include drug selection, route of administration, formulation, dosage, and duration. Drug formulations were obtained from the 2019 WHO Model List of Essential Medicines (MLEM, $1^{\text {st }}$ list) and the 2019 WHO MLEM for Children ( $7^{\text {th }}$ list); for those drug formulations that were not listed in the WHO MLEMs or where the MLEMs listed a formulation not widely available in certain African countries, drug formulations were proposed by panelists or obtained from national Essential Medicines Lists or other relevant documents. ${ }^{20,21}$

\section{Discussion}

The purpose of these first edition guidelines is to provide African healthcare workers with expert recommendations for antimicrobial prescription and use including drug selection, dosage, and duration of treatment for neonatal, pediatric, and adult patient populations to improve clinical practice and promote antimicrobial stewardship. In addition, these guidelines set forth a methodology for continuous development and updating and serve as a model for other countries and/or facilities to develop their own future national guidelines.

\section{Conclusions}

In developing these first edition guidelines and reviewing existing STGs, critical limitations and knowledge gaps emerged. First, the paucity of publicly available data on facility-level, national, or regional pathogen-specific infectious disease burden and AMR prevalence prevented these from being major considerations in infection/syndrome selection and drug selection and may represent a major limitation to their effective use. A detailed understanding of antimicrobial agent and formulation availability, including ease of access and cost considerations, at different healthcare facility levels in each AU country was also not available and may impact usability of the guidelines in certain settings. Finally, the expert panelists and external reviewers represented only a fraction of clinical care specialties and AU member states across the continent; future editions should aim to be more inclusive of non-physician clinical personnel such as nurses and community health workers.

\section{References}

1. Center for Disease Dynamics, Economic \& Policy. ResistanceMap. https://resistancemap.cddep.org/ (2021).

2. Prestinaci F, Pezzotti P, Pantosti A. Antimicrobial resistance: a global multifaceted phenomenon. Pathog Glob Health 2015;109:309-18.

3. World Health Organization. The Global Antimicrobial Resistance and Use Surveillance System (GLASS) Report: Early Implementation 2020. https://www.who.int/publicationsdetail-redirect/9789240005587 (2020).

4. Dadgostar P. Antimicrobial Resistance: Implications and Costs. Infect Drug Resist 2019;12:3903-10.

5. Peters L. et al. Multiple antibiotic resistance as a risk factor for mortality and prolonged hospital stay: A cohort study among neonatal intensive care patients with hospital-acquired infections caused by gram-negative bacteria in Vietnam. PLoS one 2019;14:e0215666.

6. Touat M. et al. Costs and Outcomes of 1 -year post-discharge care trajectories of patients admitted with infection due to antibiotic-resistant bacteria. J Infect 2021;82:339-45.

7. Akpan MR, Isemin NU, Udoh AE, Ashiru-Oredope D. Implementation of antimicrobial stewardship programmes in African countries: a systematic literature review. J Glob Antimicrob Resist 2020;22:317-24.

8. Aryee A, Price N. Antimicrobial stewardship - can we afford to do without it? Br J Clin Pharmacol 2015;79:173-81.

9. Brink AJ, et al. Antimicrobial stewardship across 47 South African hospitals: an implementation study. Lancet Infect Dis 2016;16:1017-25.

10. Charani E, et al. Investigating the cultural and contextual determinants of antimicrobial stewardship programmes across low-, middle- and high-income countries-A qualitative study. PLOS one 2019; 14:e0209847.

11. Centers for Disease Control and Prevention. The Core Elements of Hospital Antibiotic Stewardship Programs. 40 (2019).

12. Pulcini C, et al. Developing core elements and checklist items for global hospital antimicrobial stewardship programmes: a consensus approach. Clin Microbiol Infect 2019;25:20-25.

13. Wathne JS, et al. The association between adherence to national antibiotic guidelines and mortality, readmission 
and length of stay in hospital inpatients: results from a Norwegian multicentre, observational cohort study. Antimicrob Resist Infect Control 2019;8:63.

14. Craig J, Hiban K, Frost I, Kapoor G, Alimi Y, Varma JK. Comparison of national antimicrobial treatment guidelines, African Union. Bulletin of the WHO (BLT.21.286689). 2021. Available from: https://cdn.who. $\mathrm{int} / \mathrm{media} / \mathrm{docs} / \mathrm{default}$-source/bulletin/online-first/

15. Craig J, Sriram A, Hiban K, et al. The burden of antibiotic resistant bacterial pathogens among neonates and children in Africa: A systematic review and meta-analysis. Manuscr Submitt Publ 2021

16. United Nations Statistics Division. Geospatial information, Methodology. 2022. Available from: https://unstats.un.org/unsd/methodolo- gy/m49/overview/

17. Africa Centres for Disease Control and Prevention and Center for Disease Dynamics, Economics \& Policy. African Antibiotic Treatment Guidelines for Common Bacterial Infections and Syndromes- First Edition. Addis Ababa, Ethiopia. 2022. Available from: https://africaguidelines.cddep.org/download/

18. Africa Centres for Disease Control and Prevention and Center for Disease Dynamics, Economics \& Policy. African Antibiotic Treatment Guidelines for Common Bacterial Infections and SyndromesRecommended Antibiotic Treatments in Adult Patients Quick Reference Booklet. Addis Ababa, Ethiopia. 2022. Available from: https://africaguidelines.cddep.org/download/

19. Africa Centres for Disease Control and
Prevention and Center for Disease Dynamics, Economics \& Policy. African Antibiotic Treatment Guidelines for Common Bacterial Infections and Syndromes Recommended Antibiotic Treatments in Pediatric Patients- Quick Reference Booklet. Addis Ababa, Ethiopia. 2022. Available from: https://africaguidelines.cddep.org/download/

20. World Health Organization. Model List of Essential Medicines, 21st List. 1-64. 2019. Available from: https://www. who.int/publications/i/item/WHOMHP-HPS-EML-2021.02

21. World Health Organization. Model List of Essential Medicines for Children, 7th list. 2019. Available from: https://www. who.int/publications/i/item/WHOMHP-HPS-EML-2021.03 